\title{
Fuzzy parameter estimation of the Rayleigh distribution
}

\author{
T. Van Hecke \\ Ghent University, Faculty of Engineering and Architecture \\ Department of Information Technology, \\ Voskenslaan 270, 9000 Ghent, Belgium \\ E-mail: Tanja.VanHecke@ugent.be
}

\begin{abstract}
Fuzziness describes event ambiguity by means of membership functions. Probability describes the uncertainty of an event's occurrence. The primary objective of this paper is to interconnect these two disciplines by suggesting several membership function in case of the scale parameter of a Rayleigh distribution. They are based on confidence intervals and on pivotal quantities which have a normal or a $\chi^{2}$-distribution. We have succeeded in designing fuzzy numbers with a sharpness which is related to the underlying sample size. We found that a small sample size has an appreciable adverse impact on the likelihood of the parameter. We were able to improve the estimation by using upper record values.
\end{abstract}

Keywords: fuzzy sets, Rayleigh distributions, statistics

MSC 2010: 62A86, 62E86, 62G07 


\section{Introduction}

Statistical analysis, in its traditional form, is based on the crispness of data, the associated random variables, the point estimation techniques used, the hypotheses and parameters of interest and so on. On the other hand, the theory of fuzzy sets is a well established tool for the formulation and analysis of imprecise and subjective concepts [4]. Zadeh [10] states that probability must be used in concert with fuzzy logic, where both disciplines should be complementary rather than competitive. Fuzzy logic is all about degrees of truth, about fuzziness and partial or relative truths. Probability theory is interested in trying to make predictions about events from a state of partial knowledge derived from a sample. The main contribution of our paper is to show how to generate a fuzzy number from a given confidence set and therefore infer about some parameter $\theta$ under the light of fuzzy set theory. The theory of statistical inference (confidence interval (CI) and testing of hypotheses) in fuzzy environments has been discussed and developed through different approaches in the literature. Filzmoser and Viertl [2] presented an approach for statistical testing on the basis of fuzzy values by introducing the fuzzy $p$ value. Buckley [1] studied the problem of statistical inference in a fuzzy environment.

\section{Statistical distributions}

\subsection{Rayleigh distribution}

Suppose $x$ and $y$ are both Gaussian distributed (zero mean and variance $b^{2}$ ) with probability density function (pdf) $f_{G}$ as in (1).

$$
f_{G}(t)=\frac{1}{\sqrt{2 \pi} b} e^{-t^{2} /\left(2 b^{2}\right)}
$$


Consequently the radius or magnitude $r=\sqrt{x^{2}+y^{2}}$ of the complex signal $x+y I$ will be Rayleigh distributed with parameter $b$, i.e. $r: R(b)$. Here $2 b^{2}$ expresses the power of the signal and $r$ the envelope amplitude [5]. The pdf $f_{R}(r)$ described in (2) of the Rayleigh distribution is convenient for flat fading signals without lign of sight (NLOS). This distribution is a special case of the two parameter Weibull distribution with the shape parameter equal to 2 .

$$
f_{R}(r)=\frac{r}{b^{2}} e^{-r^{2} /\left(2 b^{2}\right)}
$$

The lowest order moments are

$$
\mu=\sqrt{\frac{\pi}{2}} b, \quad \sigma^{2}=\frac{4-\pi}{2} b^{2}
$$

and

$$
\text { median }=\sqrt{2 \ln (2)} b
$$

Figure 1 shows a Rayleigh distributed magnitude $r$ of a complex signal where the real and imaginary parts $x$ and $y$ are Gaussian distributed.

\section{$2.2 \quad \chi^{2}$-distribution}

Suppose $x_{i}(1 \leq i \leq k)$ are Gaussian distributed (zero mean and variance 1) and mutually independent. Then $z=\sum_{i=1}^{k} x_{i}^{2}$ is $\chi^{2}$-distributed with $k$ degrees of freedom and with $f_{\chi}$ described in (5) as pdf (Milton et al., 2013).

$$
f_{\chi}(t)=\frac{1}{2^{k / 2} \Gamma\left(\frac{k}{2}\right)} x^{\frac{k}{2}-1} e^{-x / 2}, \quad \text { for } x \geq 0
$$

with $\Gamma(z)=\int_{0}^{+\infty} t^{z-1} e^{-t} d t$. The main moments are

$$
\mu=k, \quad \sigma^{2}=2 k,
$$



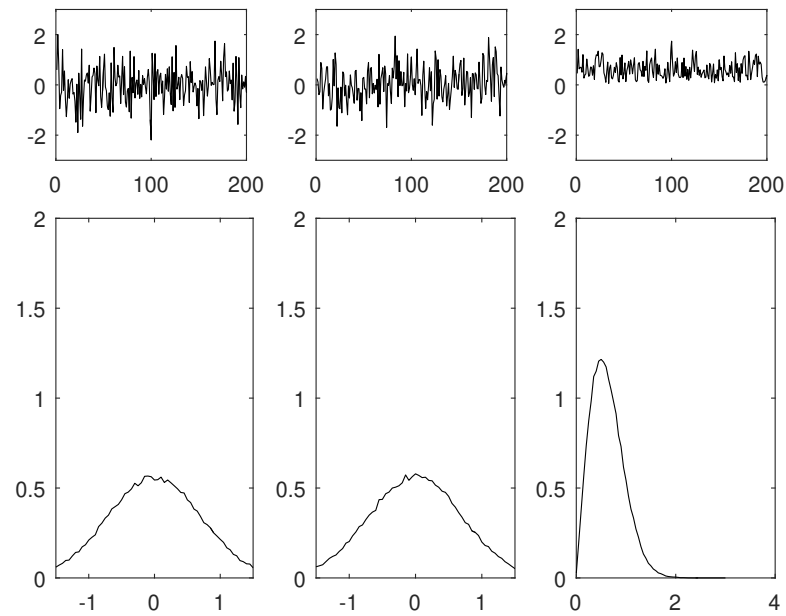

Figure 1: Rayleigh distributed variable $\sqrt{x^{2}+y^{2}}$ (at the right) out of Gaussian distributed variables $x$ and $y$ (at the left and in the middle) with the signals on top and the distributions at the bottom.

and an approximation for the median (Johnson et al., 1995; Wilson \& Hilferty, 1931) is

$$
\text { median } \approx k\left(1-\frac{2}{9 k}\right)^{3} .
$$

\section{Fuzzification}

\subsection{Fuzzy numbers}

A fuzzy number [9] can be expressed as $\tilde{n}$ where the inaccuracy is expressed by the degree of truth using a membership function $m_{\tilde{n}}$ which is at least piecewise continuous and has the functional value $m_{\tilde{n}}(x)=1$ in at least one element. The membership function reflects the extent to which $x=\tilde{n}$ i.e. $m_{\tilde{n}}(x)$ is an indicator of how likely this is. An example of a membership function is shown in Figure 2 supplemented by the $\alpha$-cut $\tilde{n}[\alpha]$ and support $s_{\tilde{n}}$ defined in (8) and 
(9) respectively.

$$
\begin{gathered}
\tilde{n}[\alpha]=\left[n_{L}(\alpha), n_{U}(\alpha)\right]=\left\{x: m_{\tilde{n}}(x) \geq \alpha\right\} \\
s_{\tilde{n}}=[l, u]=\left\{x: m_{\tilde{n}}(x)>0\right\}
\end{gathered}
$$

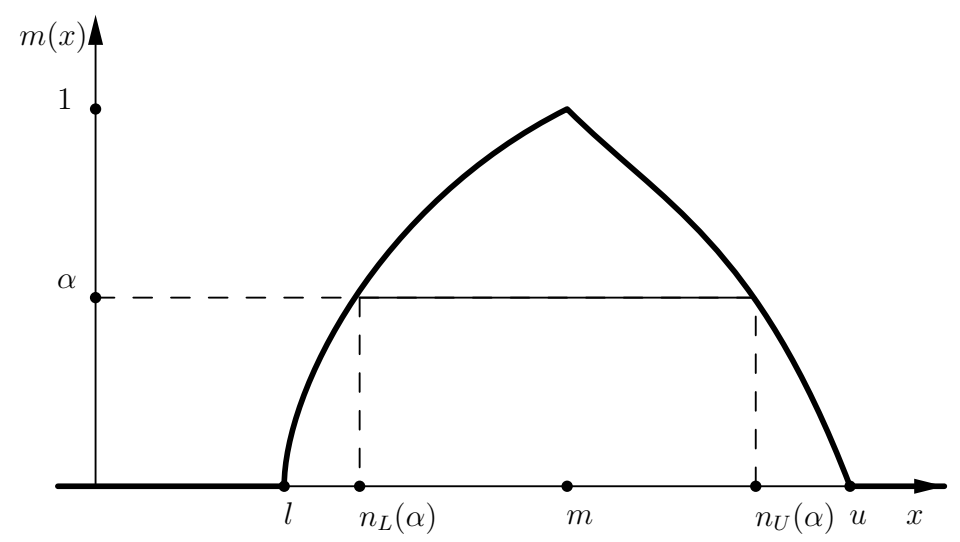

Figure 2: Degree of membership function with $\alpha$-cut $\left[n_{L}(\alpha), n_{U}(\alpha)\right]$ and support $[l, u]$.

\subsection{Fuzzy parameter estimates}

Buckley [1] introduced a technique to derive explicit membership functions for parameters of a distribution. Assume that $\theta$ is a unknown parameter to be estimated. Based on a sample one can pbtain a set of $(1-\beta) 100 \%$ confidence intervals $\left[\theta_{L}(\beta), \theta_{U}(\beta)\right]$ for $0 \leq \beta \leq 1$ using a pivotal quantity [7] $Q$ with a known distribution. The membership function of the fuzzy number $\tilde{\theta}$ is constructed by stacking these confidence intervals for different $\beta$-values one above the other. In this manner the $\alpha$-cuts of $\tilde{\theta}$ are the confidence intervals for a confidence of $1-\alpha$.

If $u \in \tilde{\theta}[\alpha]$, then $\theta_{L}(\alpha) \leq u \leq \theta_{U}(\alpha)$, i.e. $P\left(\theta_{L}(\alpha) \leq \theta \leq \theta_{U}(\alpha)\right)=1-\alpha$. This is equivalent to $F_{D}(Q)=1-\frac{\alpha}{2}$ if a pivotal quantity $Q$ with distribution 
$D$ is used to derive the CI. Its cumulative distribution function (cdf) is $F_{D}$ with median $M e$. As a consequence the membership function for $\theta$ can be written as

$$
m_{D}(u)= \begin{cases}2-2 F_{D}\left(Q_{u}\right), & \text { if } 0 \leq u<Q^{-1}(M e) \\ 2 F_{D}\left(Q_{u}\right), & \text { if } u \geq Q^{-1}(M e)\end{cases}
$$

where $Q_{u}$ is the value of the pivotal quantity $Q$ for $\theta=u$. We will suggest 3 approaches based on different pivotal quantities. The following theorem bridges the gap between a sample for a Rayleigh distributed variable and a pivotal quantity with a $\chi^{2}$-distribution.

Theorem: Suppose $\left\{r_{i} \mid 1 \leq i \leq n\right\}$ is a sample from a Rayleigh distribution with parameter $b$, then $\frac{n \overline{r^{2}}}{b^{2}}$ has a $\chi^{2}$-distribution with $2 n$ degrees of freedom.

Proof: As a consequence of the definition of the Rayleigh distribution, $\frac{r_{i}^{2}}{b^{2}}$ has a $\chi^{2}$-distribution with 2 degrees of freedom for each sample value $r_{i}(1 \leq i \leq n)$ out of a $R(b)$. This makes that $\frac{n \overline{r^{2}}}{b^{2}}$, with $\overline{r^{2}}=\frac{1}{n} \sum_{i=1}^{n} r_{i}^{2}$, has a $\chi^{2}$-distribution with $2 n$ degrees of freedom.

\section{$3.3 \quad \chi^{2}$-approach}

If $\chi_{p}^{2}(\nu d . f$.$) is the critical value with a probability of p$ of lying on its left, related to a $\chi^{2}$-distribution with $\nu$ degrees of freedom, a $(1-\beta) 100 \%$ confidence interval for $b^{2}$ can be written as

$$
\left[\frac{n \overline{r^{2}}}{\chi_{1-\beta / 2}^{2}(2 n d . f .)}, \frac{n \overline{r^{2}}}{\chi_{\beta / 2}^{2}(2 n d . f .)}\right]
$$

Based on theorem 1, we suggest the membership function $m_{\chi}(u)$ for an estimation $u$ of $b$ which is equal to

$$
m_{\chi}(u)= \begin{cases}2-2 F_{\chi, 2 n}\left(\frac{n \overline{r^{2}}}{u^{2}}\right), & \text { if } 0 \leq u<\sqrt{\frac{n \overline{r^{2}}}{M e}} \\ 2 F_{\chi, 2 n}\left(\frac{n \overline{r^{2}}}{u^{2}}\right), & \text { if } u \geq \sqrt{\frac{n \overline{r^{2}}}{M e}}\end{cases}
$$




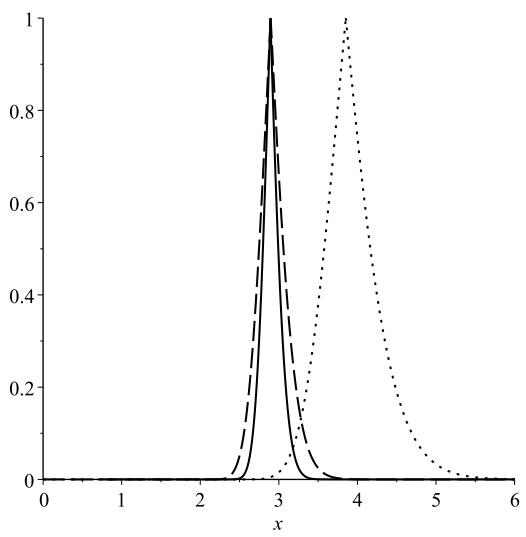

Figure 3: Degree of membership function of parameter $b$ from a sample out of a $R(3)$ distribution with the $\chi^{2}$-approach.

$(n \in\{20$ (dot), 40 (dash), 100 (solid) $\})$

and can be found in Figure 3 for a sample from $R(3)$. Here $F_{\chi, \nu}$ is the cdf of a $\chi^{2}$-distribution with $\nu$ degrees of freedom. Wilson and Hilferty [8] came up with the idea of transforming a $\chi^{2}$ variable in order to construct a Gaussian approximation for it by accelerating its convergence to normality. Figure 3 shows clearly that the median is badly estimated in case of small $n$-values.

\subsection{Gaussian asymptotic approach}

As the median of a $\chi^{2}$-distribution is difficult to estimate, especially for small sample values, an improved approach is required. As theorem 1 states that $\frac{n \overline{r^{2}}}{b^{2}}$ has a $\chi^{2}$-distribution with $2 n$ degrees of freedom, this variable can be approximated by a normal distribution with mean $(2 n)$ and variance $(4 n)$ [7]. This method gives rise to an alternative membership function as in (14) with $F_{N}$ the cumulative distribution function of Gaussian distribution. The point estimator $\hat{b}=\sqrt{\frac{\bar{r}}{2}}$ is a consequence of (6) and theorem 1. The standardization 


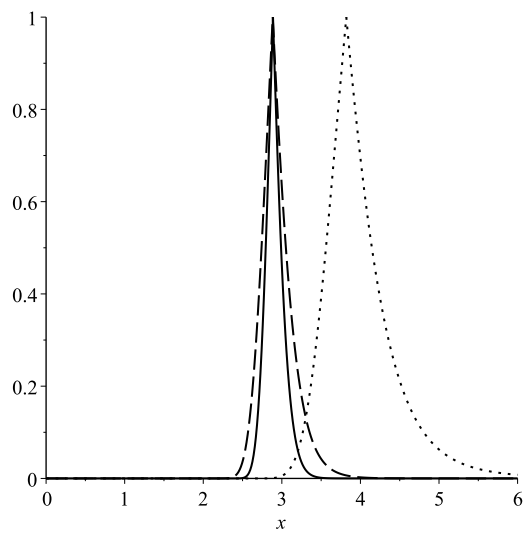

Figure 4: Degree of membership function of parameter $b$ from a sample out of a $R(3)$ distribution with the Gaussian approach. $(n \in\{20$ (dot), 40 (dash), 100 (solid) $\})$

of the Gaussian variable is explained in (14).

$$
\begin{aligned}
& \frac{n \overline{r^{2}}}{b^{2}}: N(2 n, \sqrt{4 n}) \\
& \Rightarrow \frac{\frac{n \overline{r^{2}}}{b^{2}}-2 n}{2 \sqrt{n}}=\sqrt{n}\left(\frac{\overline{r^{2}}}{2 b^{2}}-1\right): N(0,1)
\end{aligned}
$$

Figure 4 shows that the sharpness of the fuzzy number $\tilde{\theta}$ is improved when the underlying sample is larger.

$$
m_{N}(u)= \begin{cases}2-2 F_{N}\left(\sqrt{n}\left(\frac{\overline{r^{2}}}{2 u^{2}}-1\right)\right), & \text { if } 0 \leq u<\sqrt{\frac{r^{2}}{2}} \\ 2 F_{N}\left(\sqrt{n}\left(\frac{\overline{r^{2}}}{2 u^{2}}-1\right)\right), & \text { if } u \geq \sqrt{\frac{r^{2}}{2}}\end{cases}
$$

\subsection{Upper Record Values}

As the popular maximum likelihood method suffers from bias in case of small sample sizes, Seo [6] suggest exact confidence intervals for the parameter $b$ of 
the Rayleigh distribution based on upper record values.

Definition:Let $x_{1}, x_{2}, \ldots, x_{n}$ be a sequence of $n$ independent sample values from the same distribution. If $x_{j}>x_{i}$ for all $i<j$, then $x_{j}$ is an upper value record. The indices where the upper values occur, are the record times $\{R T(k), k \geq 1\}$, with $R T(1)=1$ and $R T(k)=\min \left\{j \mid j>R T(k-1), x_{j}>\right.$ $x_{R T(k-1)}$.

Based on the spacing and with $I$ the largest record time of a sample from a Rayleigh distribution with parameter $b$, a pivotal quantity (15) is obtained:

$$
\frac{x_{R T(I)}^{2}}{b^{2}}: \quad \chi^{2}(2 I d . f .),
$$

which enables us to construct membership function (16)

$$
m_{U R V}(u)= \begin{cases}2-2 F_{\chi, 2 I}\left(\frac{x_{R T(I)}^{2}}{u^{2}}\right), & \text { if } 0 \leq u<\hat{u} \\ 2 F_{\chi, 2 I}\left(\frac{x_{R T(I)}^{2}}{u^{2}}\right), & \text { if } u \geq \hat{u}\end{cases}
$$

shown in Figure 5 for a point estimator $\hat{u}_{M e}=\frac{x_{R T(I)}}{\sqrt{M e}}$ for $b$ which is based on the median (dot) and for a point estimator $\hat{u}_{\bar{x}}=\frac{x_{R T(I)}}{\sqrt{2 I}}$ which is based on the mean (dash). Here $M e=2 I\left(1-\frac{1}{9 I}\right)^{3}$, based on (7). The use of $\hat{u}_{\bar{x}}$ tends to suffer from discontinuity problems for the membership function. The upper value record approach is mostly helpful and superior to the traditional $\chi^{2}$-approach (solid) for small samples as can be seen by comparing Figure 5 and Figure 6 .

\section{Conclusions}

We have shown how fuzziness can benefit from probability knowledge. The degree of truth about the parameter value of the frequently used Rayleigh distribution for the envelope of a received signal, is expressed by its membership 


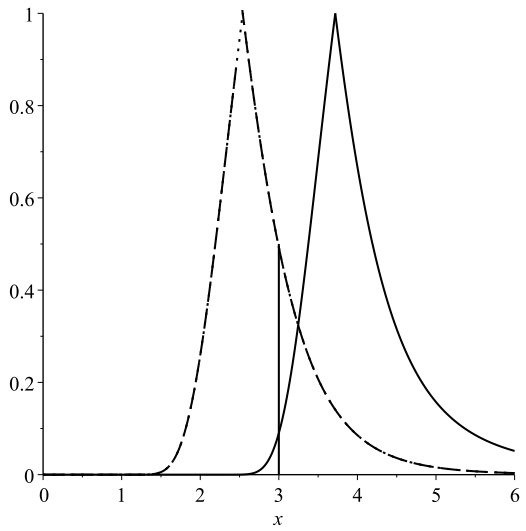

Figure 5: Degree of membership function of parameter $b$ from a sample out of a $R(3)$ distribution with the upper record value $(n=10)$, with point estimator based on the median (dot) or on the mean (dash) and with the traditional $\chi^{2}$-approach (solid).

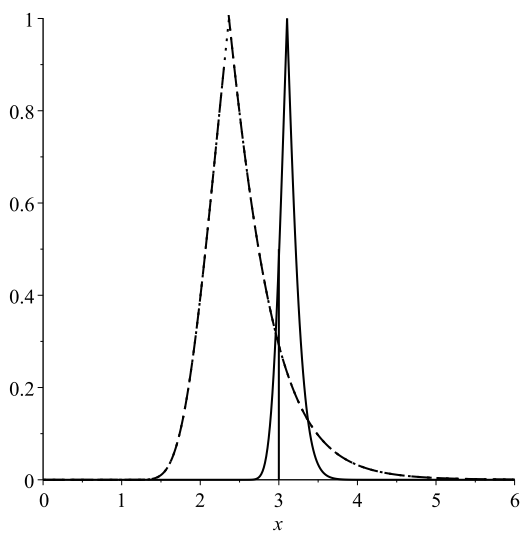

Figure 6: Degree of membership function of parameter $b$ from a sample out of a $R(3)$ distribution with the upper record value $(n=100)$, with point estimator based on the median (dot) or on the mean (dash) and with the traditional $\chi^{2}$-approach (solid). 
function. We explained how valuable functions can be developed, based on confidence intervals, samples and pivotal quantities. We noticed that the quality of the point estimator is superior in case of the Gaussian distribution. We showed that switching to upper record values, leads to results that are sharper and less biased in case of small sample sizes.

\section{References}

[1] J.J. Buckley, Fuzzy statistics: hypothesis testing, Soft Computing 9 (2005), 512-518.

[2] P. Filzmoser and R. Viertl, Testing of hypotheses with fuzzy data: the fuzzy p-value, Metrika 59 (2004), 21-29.

[3] N.L. Johnson, S. Kotz and N. Balkrishnan, Continuous univariate distributions, 2nd ed. New York: John Wiley and Sons, 1995.

[4] B. Kosko, Fuzziness vs. probability, Internatial Journal of General Systems, 17 (1990), 211-240.

[5] J.S. Milton, J.C. Arnold and K.I. Liu, Probability and Statistics: For Engineering and the Computing Sciences, McGraw Hill, 2013.

[6] J. Seo, J. Jeon and S. Kang, Exact interval inference for the two-parameter Rayleigh distribution based on the upper record values, Journal of Probability and Statistics, article ID 8246390 (2016), 1-5.

[7] J. Shao, Mathematical Statistics, New York: Springer-Verlag, 2003.

[8] E.B. Wilson and M.M. Hilferty, The distribution of chi-square, Proceedings of the National Academy of Sciences of the United States of America $\mathbf{1 7}$ (1931), 684-688. 
[9] L.A. Zadeh, Fuzzy sets, Information and Control 8 (3) (1965), 338-353.

[10] L.A. Zadeh, Discussion: Probability Theory and Fuzzy Logic Are Complementary Rather Than Competitive, Technometrics 37 (1995), 271-276. 